\title{
Power System Stabilizer Driven by an Adaptive Fuzzy Set for Better Dynamic Performance
}

\author{
H. F. Soliman, A.-F. Attia, M. Hellal, M. A. L. Badr
}

This paper presents a novel application of a fuzzy logic controller (FLC) driven by an adaptive fuzzy set (AFS) for a power system stabilizer (PSS).The proposed FLC, driven by AFS, is compared with a classical FLC, driven by a fixed fuzzy set (FFS). Both FLC algorithms use the speed error and its rate of change as input vectors. A single generator equipped with FLC-PSS and connected to an infinite bus bar through double transmission lines is considered. Both FLCs, using AFS and FFS, are simulated and tested when the system is subjected to different step changes in the reference value. The simulation results of the proposed FLC, using the adaptive fuzzy set, give a better dynamic response of the overall system by improving the damping coefficient and decreasing the rise time and settling time compared with classical FLC using FFS. The proposed FLC using AFS also reduces the computational time of the FLC as the number of rules is reduced.

Keywords: fuzzy controller: static and adapted fuzzy sets and power system stabilizer.

\section{Introduction}

The design of a power system stabilizer is an important issue from the viewpoint of power system stability, since it damps out local plant modes and inter area modes of oscillation without compromising the stability of other modes. A conventional power system stabilizer (CPSS) comprises of a wash out circuit and a cascade of two phase lead networks. To design such a PSS, many strategies [1-6] have been proposed that included techniques such as root locus, Bode plots, eigenvalue assignment, multivariable frequency domain techniques, optimal control design, etc. New techniques [7-10] based on expert systems, neural networks and rule based fuzzy logic for PSS design is also emerging.

The fuzzy logic controller has emerged as a very active and fruitful research area. The adaptive fuzzy controllers provide a means for continuously adapting the fuzzy set to match the desired performance criteria. Its typical application area is for controlling time varying and/or nonlinear plants. Fuzzy controllers may be either static or dynamic. The static fuzzy rules are usually based on operator experience, as fuzzy logic can easily encode linguistic information [11]. This is the main advantage of the fuzzy logic controller over neural networks. In the adaptive case, the linguistic information captured from operator experience can be used to initialize the fuzzy set. This helps to reduce the number of iterations in the training [12].

In this paper, three fuzzy controllers are proposed for PSS. The first uses seven fixed fuzzy sets for the input and output variables. The second uses five fixed fuzzy sets for the input and output variables. The third controller is proposed as an adaptation scheme, based on the back propagation (BP) algorithm [12], which dynamically varies the fuzzy sets to achieve better dynamic performance. The performance of PSS with both static and adaptive fuzzy logic controllers is compared through the simulation results, when the system is subjected to various disturbances.

\section{System under study and a mathematical model of it}

The system under study consists of a single synchronous generator (S.G) connected to the infinite bus via double short transmission lines, as shown in Fig. 1. A Matlab program has been developed for the system under study. The synchronous generator is equipped with an automatic voltage regulator (AVR). The equations of the system are derived in the $d$ and $q$-axis in $[13,14]$. The magnetic circuit is assumed to be linear. The differential equations that describe the system under study are as follows:

$\Delta \omega^{\prime}=\frac{\Delta T_{m}-D \times \Delta \omega-K_{1} \times \Delta \delta-K_{2} \times \Delta e_{q}^{\prime}}{M}$

$\Delta \delta^{\prime}=\omega_{b} \times \Delta \omega$

$\Delta \Delta e_{q}^{\prime}=\frac{\Delta E_{F D}-K_{4} \times \Delta \delta}{T_{d o}^{\prime}}-\frac{\Delta e_{q}^{\prime}}{T_{d o}^{\prime} \times K_{3}}$

$\Delta E_{F D}^{\prime}=\frac{K_{A} \times \Delta V_{t}-K_{A} \times K_{5} \times \Delta \delta-K_{A} \times K_{6} \times \Delta e_{q}^{\prime}}{T_{A}}+$

$$
-\frac{\Delta E_{F D}+K_{A} \times U_{p s s}}{T_{A}}
$$

where: $\Delta$ defines a small change in the next symbol or variable, $\omega$ is the mechanical angular speed, $T_{m}$ is the mechanical torque. $\delta$ defines the power angle and $\omega_{b}$ is the base angular speed. $e_{q}^{\prime}$ is the voltage behind the transient reactance in the $d$-axis. The equations which describe the constants $K_{1}$ to $K_{6}$ and variable $E_{F D}$ are given in $[13,14] . T_{d o}^{\prime}$ is the field winding open circuit time constant (sec), $V_{t}$ is the terminal voltage. $K_{A}$ and $T_{A}$ are the exciter gain and time constant. The values of $K_{A}$ and $T_{A}$ are given in Appendix $1, U_{p s s}$ is the output of the power system stabilizer. All the system parameters and constants are given in Appendix 1. The mathematical equations of the AVR together with the exciter and the governor are given as follows: 


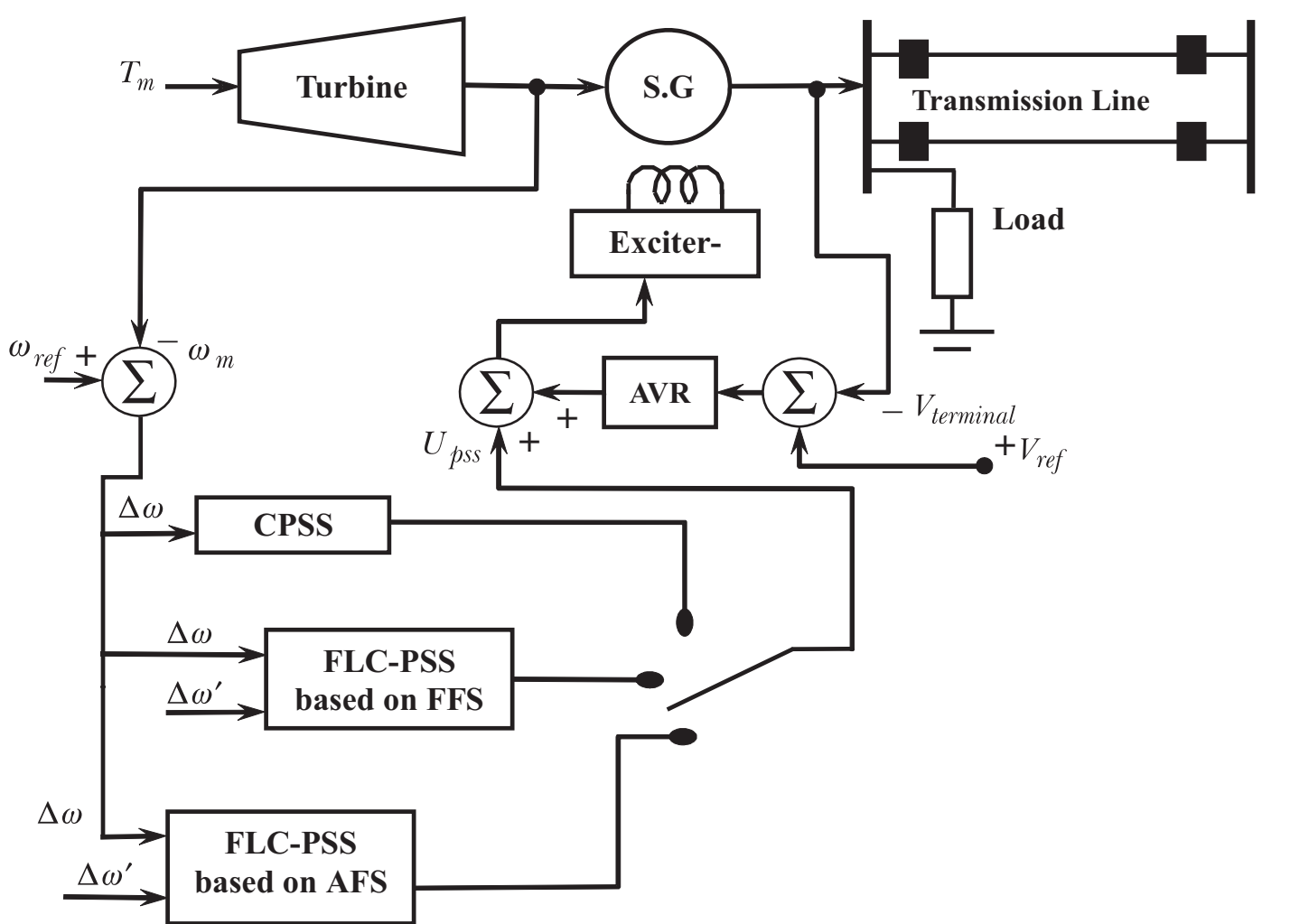

Fig. 1: The synchronous generator, equipped with FLC-PSS based on FFS or AFS or CPSS

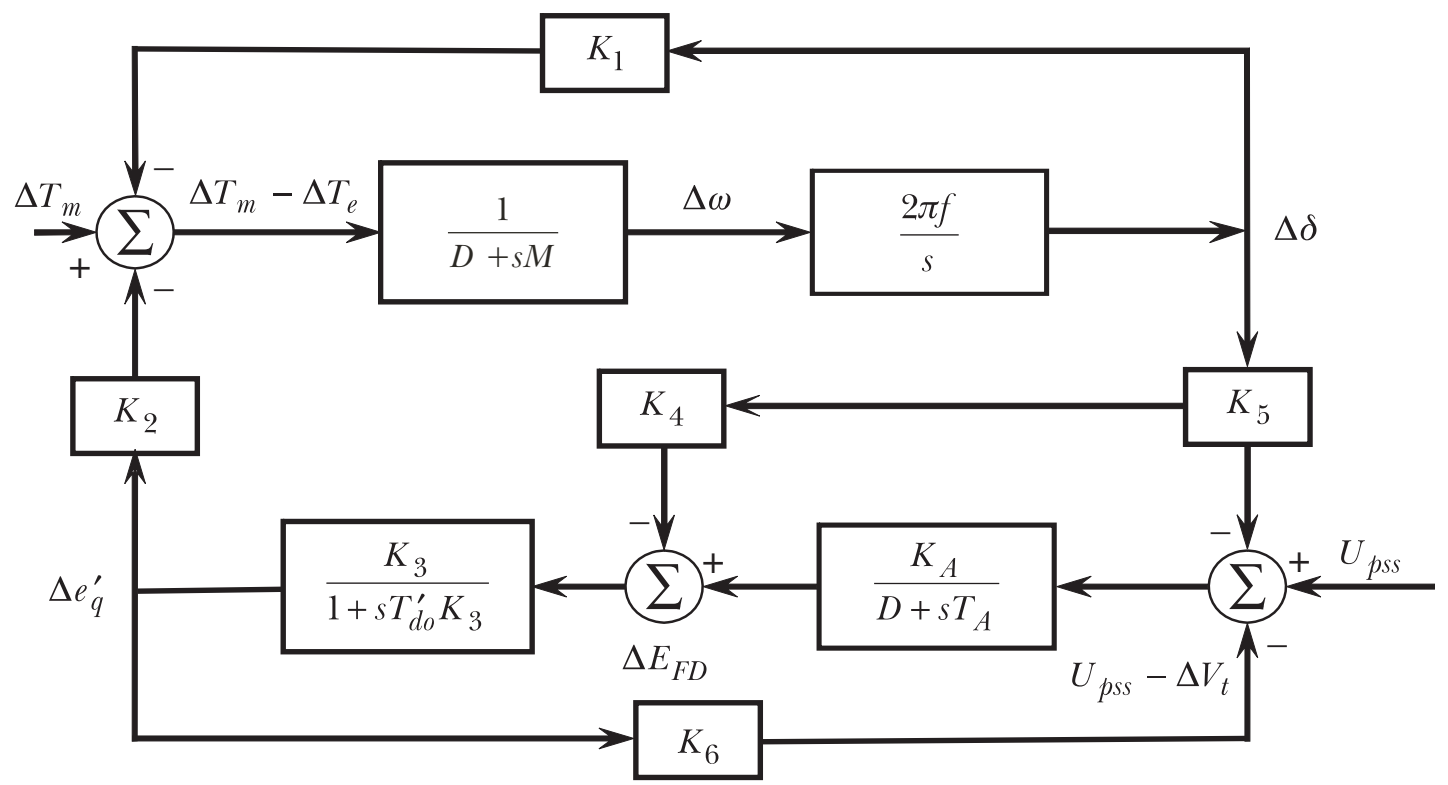

Fig. 2: Transfer function block diagram

$E_{F D}=\left(\frac{K_{A}}{1+s T_{A}}\right)\left(V_{r e f}-V_{t}+U_{p s s}\right)$,

$g=\left(\left(a+\frac{b}{1+s T_{g}}\right) \times \dot{\delta}\right)$

where $V_{\text {ref }}$ is the reference terminal voltage, $a$, and $b$ are constants, $T_{g}$ is the governor time constant. Their values are given in Appendix 1. The complete system model, in block diagram presentation, is shown in Fig. 2.
All the system parameters and the constants of AVR, exciter, governor, and conventional PSS are given in Appen$\operatorname{dix} 1$.

\section{Fuzzy logic controller}

Fuzzy control systems are rule-based systems. A set of fuzzy rules represents the FLC mechanism to adjust the effect of certain system stimuli. The aim of fuzzy control systems is normally to replace a skilled human operator with a fuzzy rules-based system. Also the FLC provides an algorithm which can convert the linguistic control strategy, based on 


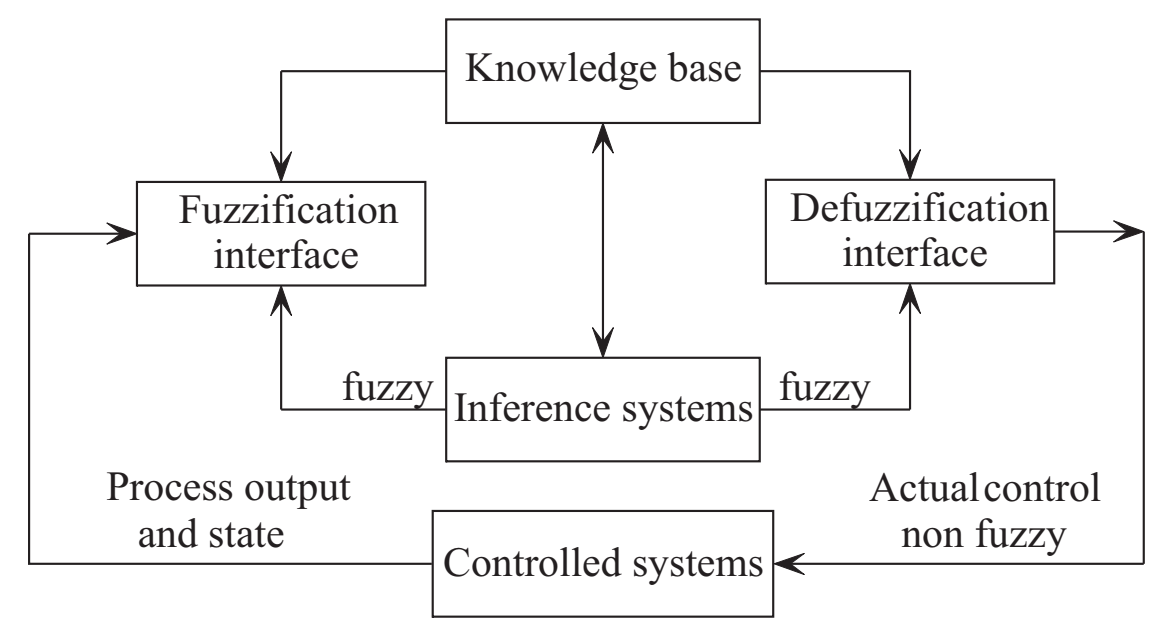

Fig. 3: Generic structure of fuzzy logic controller

expert knowledge, to automatic control strategies. Fig. 3 depicts the basic configuration of the FLC. It consists of a

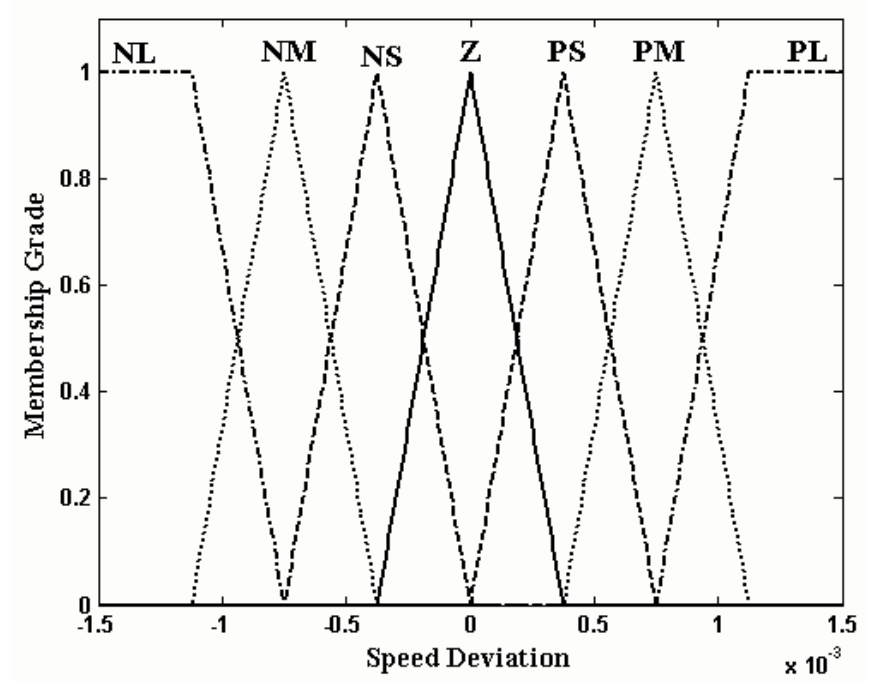

Fig. 4: Input fuzzy sets for speed deviation

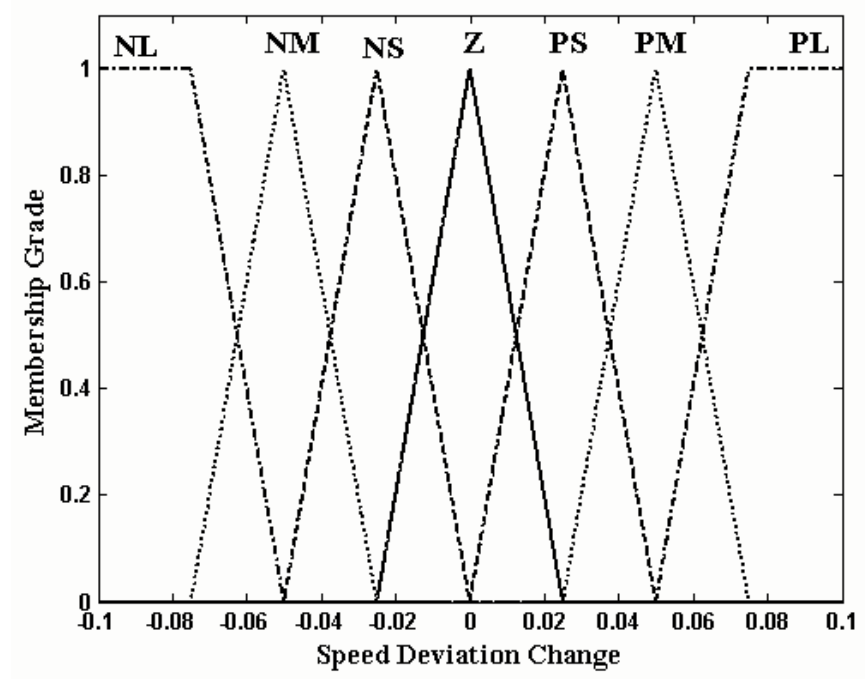

Fig. 5: Input fuzzy sets for speed deviation change fuzzification interface, a knowledge base, decision making logic, and a defuzzification interface [11].

\subsection{Global input variables}

The fuzzy input vector consists of two variables; first, the generator speed deviation $\Delta \omega$ and, second, the acceleration $\Delta \omega^{\prime}$. We designed two fuzzy controllers; the first is the fuzzy controller with seven linguistic variables, using fixed fuzzy sets for each one of the input variables, as shown in Fig. 4 and Fig. 5, respectively. The fuzzy set for the output variable is shown in Fig. 6. In these figures, linguistic variables have been used such as PL (Positive Large), PM (Positive Medium), PS (Positive Small), Z (Zero), NS (Negative Small), NM (Negative Medium), NL (Negative Large). They have been used for the output fuzzy set, adding linguistic $\mathrm{U}$ to the related ones, as indicated in Table 1.

The second fuzzy controller, based on five linguistic variables, is used for each input variable, as shown in Fig. 7 and Fig. 8. respectively. The fuzzy set for the output variables is shown in Fig. 9. The fuzzy sets shown in Figs. 7, 8, 9 are used for both FLC-PSS with a fixed and adaptive fuzzy set. The solid line, in these figures, represents FFS, while the dotted lines depict these fuzzy sets after applying the adaptation techniques via the back propagation algorithm [12]. The

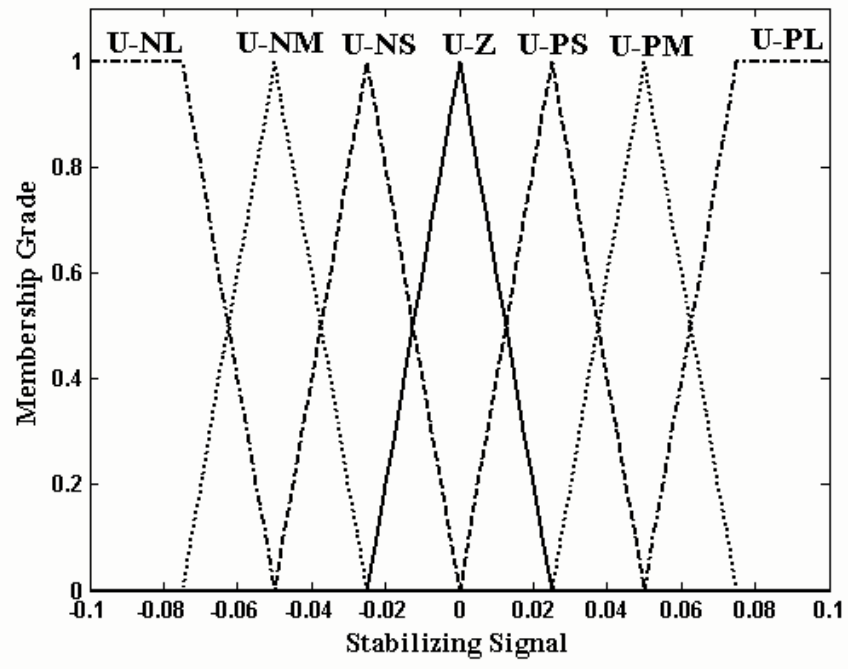

Fig. 6: Output fuzzy sets 


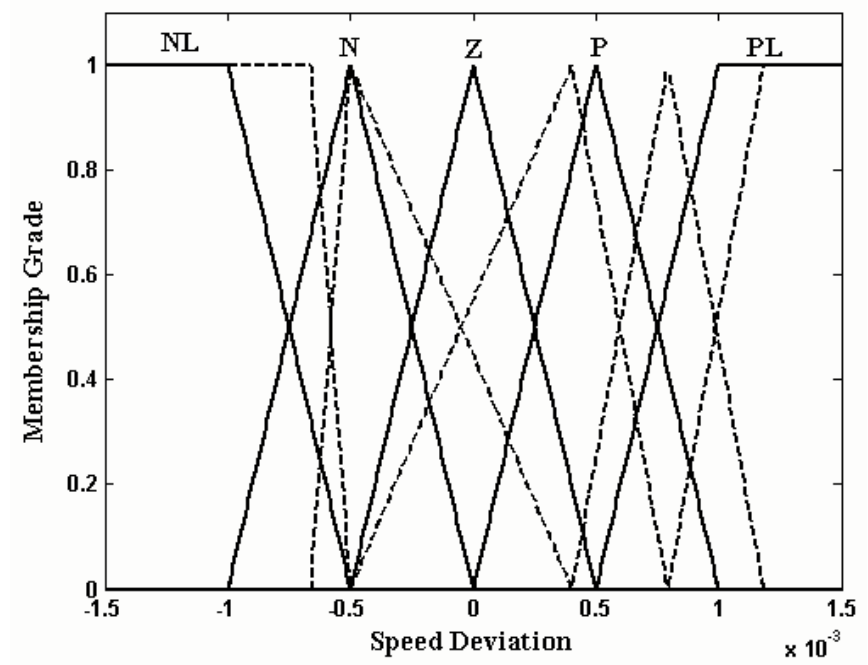

Fig. 7: Solid lines: represent the FFS or MFs before adaptation. Dashed lines: represent the AFS or Normalized MFs after adaptation.

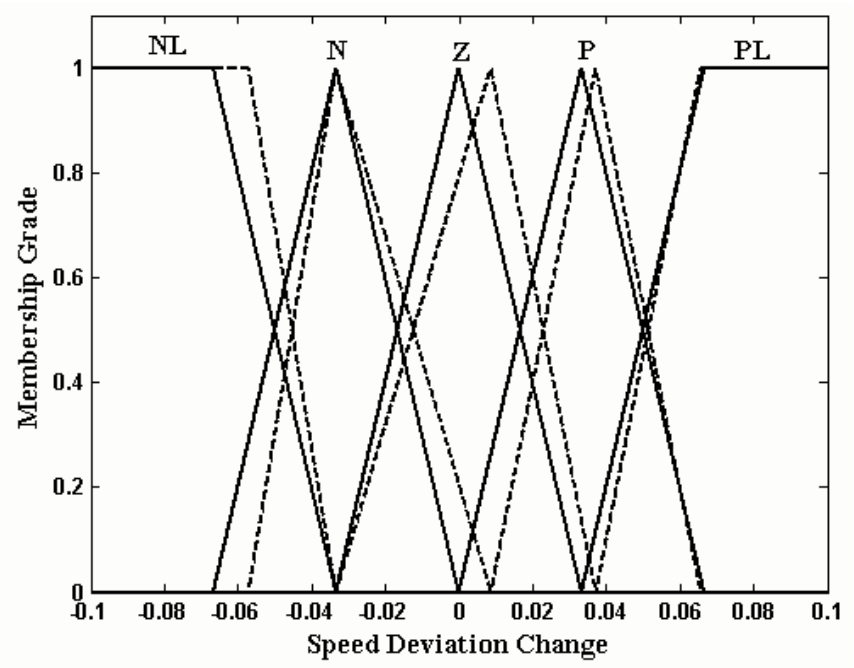

Fig. 8: Solid lines: represent the FFS or MFs before adaptation. Dashed lines: represent the AFS or Normalized MFs after adaptation.

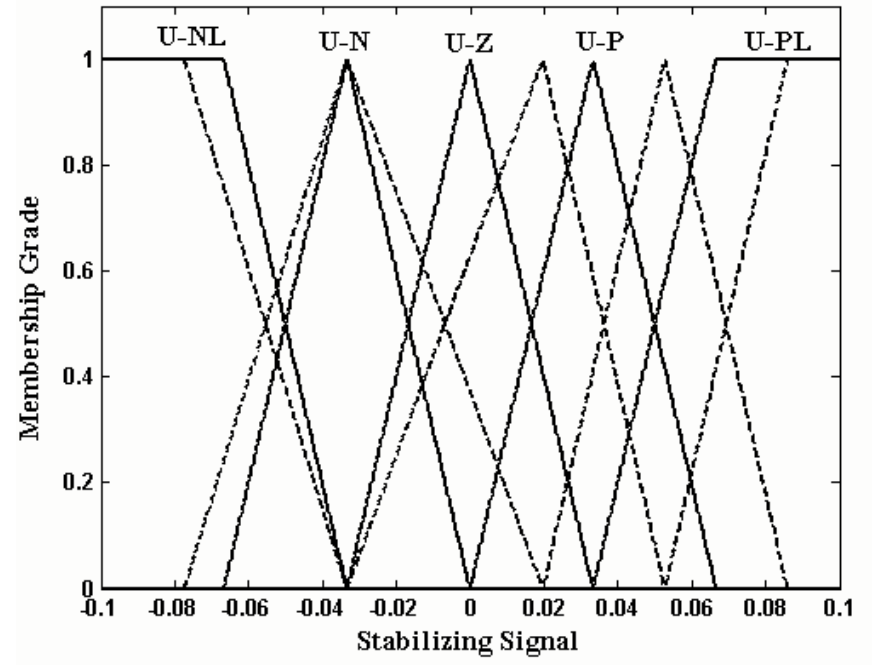

Fig. 9: Solid lines: represent the FFS or MFs before adaptation. Dashed lines: represent the AFS or Normalized MFs after adaptation.

adaptation technique is used to modify the membership function $(M F)$ of each fuzzy set. In these figures, $M F s$ of the linguistic variables have been used, such as PL (Positive Large), P (Positive), Z (Zero), N (Negative), NL (Negative Large). These linguistic variables have been used for the output fuzzy set, adding linguistic $\mathrm{U}$ to the related ones, as indicated in Table 2.

Generally, after building up the fuzzy set for input and output variables it is required to develop the set of rules, the so-called Look-up Table, in which the relation between the input variable, $\Delta \omega$ and $\Delta \omega^{\prime}$, and the output variable of fuzzy controller is defined. The output of the FLC is used to adjust the $U_{p s s}$ value, as shown in Fig. 1. These look-up tables are given in Table 1, when using seven fuzzy sets (7FLC), and Table 2, when using five fuzzy sets (5FLC).

The surface viewer, as shown in Fig. 10, has a special capability that is very helpful in cases with two (or more) inputs and one output. This figure shows the output surface of the FLC of the system versus two inputs.

Table 1: Look-up table defining the relation between the input and output variable in a fuzzy set form for seven fuzzy sets

\begin{tabular}{|c|c|c|c|c|c|c|c|}
\hline $\begin{array}{c}\text { Speed deviation } \\
(\Delta \omega)\end{array}$ & \multicolumn{7}{|c|}{$\begin{array}{c}\text { Speed deviation chang } \\
\left(\Delta \omega^{\prime}\right)\end{array}$} \\
\hline & NL & NM & NS & Z & PS & PM & PL \\
\hline NL & U_NL & U_NL & U_NL & U_NL & U_NM & U_PS & U_Z \\
\hline NM & U_NL & U_NM & U_NM & U_NM & U_NS & U_Z & U_PS \\
\hline NS & U_NL & U_NM & U_NS & U_NS & U_Z & U_PS & U_PM \\
\hline Z & U_NL & U_NM & U_NS & U_Z & U_PS & U_PM & U_PL \\
\hline PS & U_NM & U_NS & U_Z & U_PS & U_PS & U_PM & U_PL \\
\hline PM & U_NS & U_Z & U_PS & U_PM & U_PM & U_PL & U_PL \\
\hline PL & U_Z & U_PS & U_PM & U_PL & U_PL & U_PL & U_PL \\
\hline
\end{tabular}


Table 2: Look-up table defining the relation between the input and output variable in a fuzzy set form for five fuzzy sets

\begin{tabular}{|c|c|c|c|c|c|}
\hline \multirow{2}{*}{ Speed deviation $(\Delta \omega)$} & \multicolumn{5}{|c|}{ Speed deviation chang $\left(\Delta \omega^{\prime}\right.$ t $)$} \\
\cline { 2 - 6 } & $\mathrm{NL}$ & $\mathrm{N}$ & $\mathrm{Z}$ & $\mathrm{P}$ & $\mathrm{PL}$ \\
\hline $\mathrm{NL}$ & $\mathrm{U}-\mathrm{NL}$ & $\mathrm{U}-\mathrm{NL}$ & $\mathrm{U}-\mathrm{NL}$ & $\mathrm{U}-\mathrm{N}$ & $\mathrm{U}-\mathrm{Z}$ \\
\hline $\mathrm{N}$ & $\mathrm{U}-\mathrm{NL}$ & $\mathrm{U}-\mathrm{NL}$ & $\mathrm{U}-\mathrm{N}$ & $\mathrm{U}-\mathrm{Z}$ & $\mathrm{U}-\mathrm{P}$ \\
\hline $\mathrm{Z}$ & $\mathrm{U}-\mathrm{NL}$ & $\mathrm{U}-\mathrm{N}$ & $\mathrm{U}-\mathrm{Z}$ & $\mathrm{U}-\mathrm{P}$ & $\mathrm{U}-\mathrm{PL}$ \\
\hline $\mathrm{P}$ & $\mathrm{U}-\mathrm{N}$ & $\mathrm{U}-\mathrm{Z}$ & $\mathrm{U}-\mathrm{P}$ & $\mathrm{U}-\mathrm{PL}$ & $\mathrm{U}-\mathrm{PL}$ \\
\hline $\mathrm{PL}$ & $\mathrm{U}-\mathrm{Z}$ & $\mathrm{U}-\mathrm{P}$ & $\mathrm{U}-\mathrm{PL}$ & $\mathrm{U}-\mathrm{PL}$ & $\mathrm{U}-\mathrm{PL}$ \\
\hline
\end{tabular}

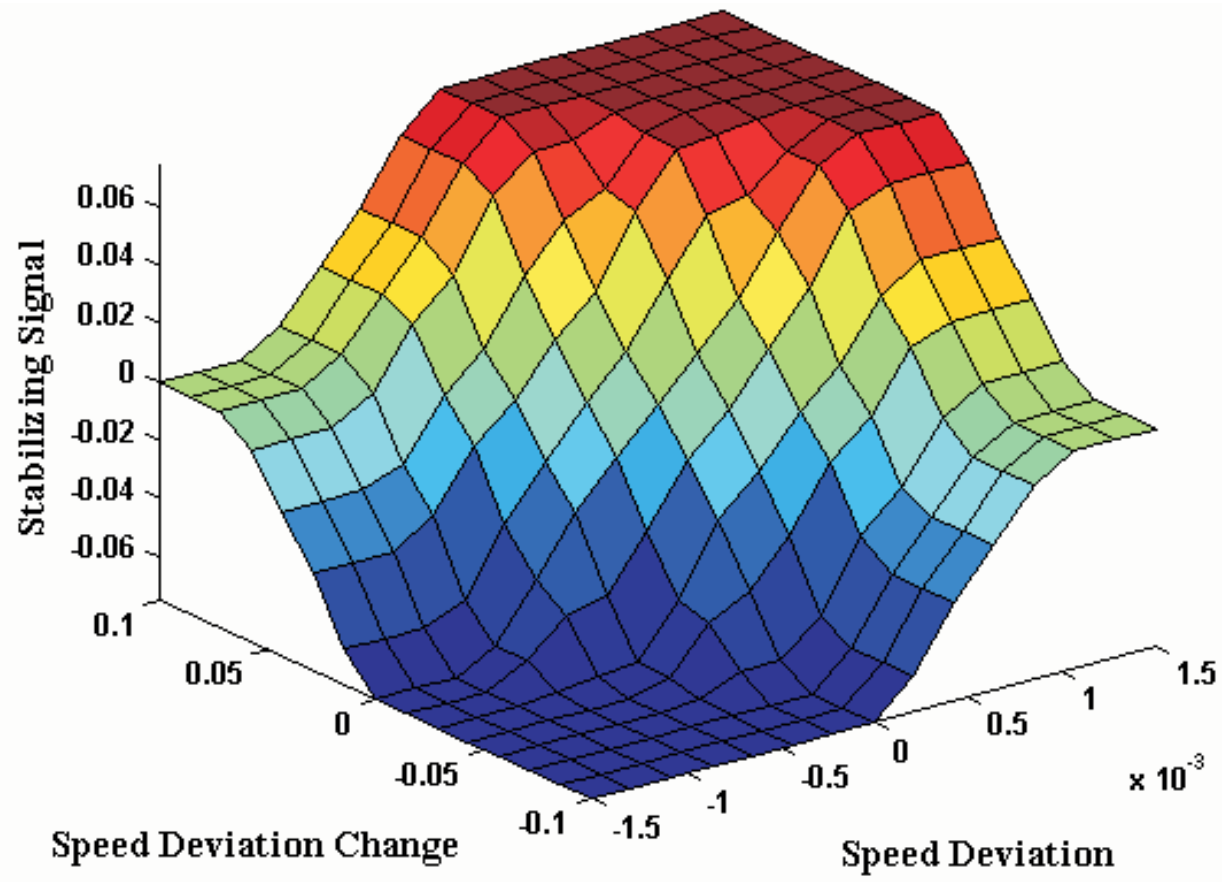

Fig. 10: Rules surface viewer for FLC using a fuzzy set

\subsection{The defuzzification method}

The Minimum of Maximum value method has been used to calculate the output from the fuzzy rules. This output is usually represented by a polyhedron map, as shown in Fig. 11. The defuzzification stage is executed in two steps. First, the minimum membership is selected from the minimum value of the two input variables $\left(x_{1}\right.$ and $\left.x_{2}\right)$ with the related fuzzy set

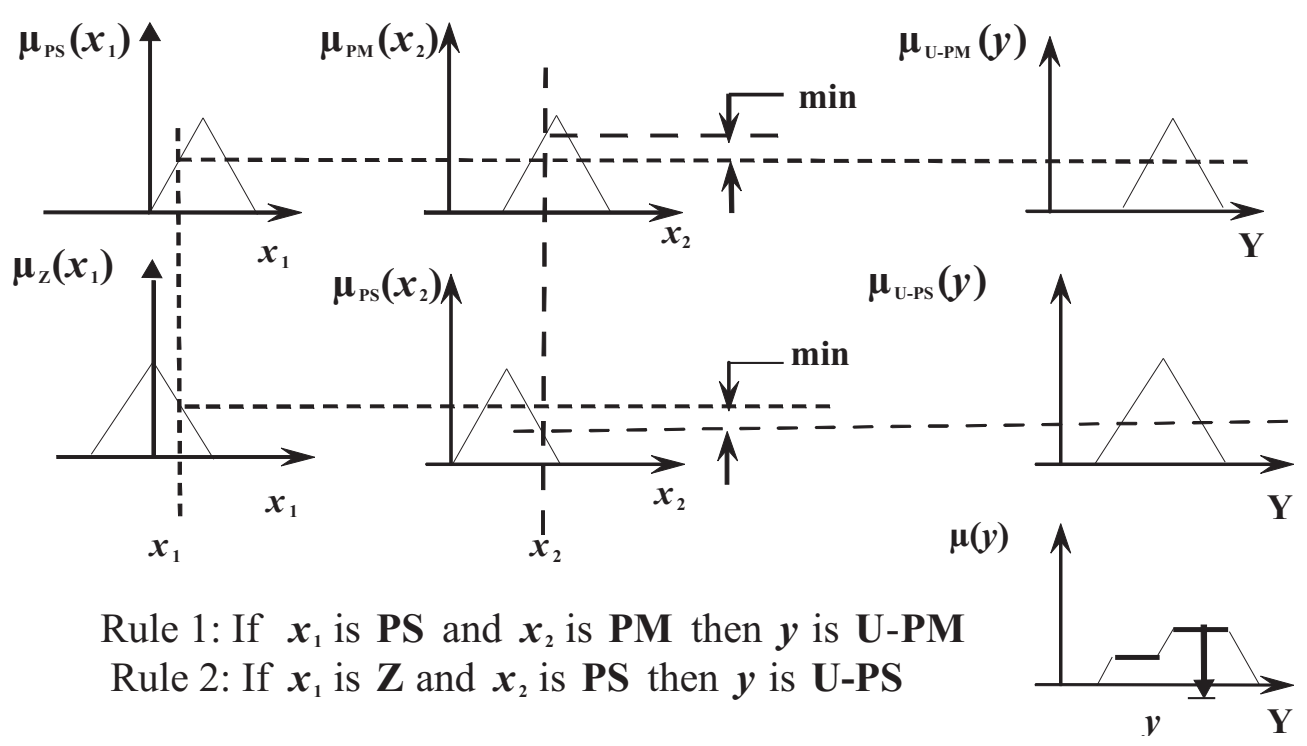

Fig. 11: Schematic diagram of the defuzzification method using the center of area 
in that rule. This minimum membership is used to rescale the output rule, and then the maximum is taken to give the final polyhedron map, as shown in Fig. 11. Finally, the centroid or center of area has been used to compute the fuzzy output, which represents the defuzzification stage, as follows:

$$
U_{p s s}=\frac{\int y \mu(y) \mathrm{d} y}{\int \mu(y) \mathrm{d} y}
$$

\section{The proposed adaptive fuzzy logic controller}

The adaptive fuzzy logic controller (AFLC), using an adaptive fuzzy set, has the same inputs and output as a static FLC. A full rule base (25 rules) is also defined. The rules have the general form:

If $\Delta \omega$ is $\mathbf{N L}$ and $\Delta \omega^{\prime}$ ţ is $\mathbf{N}$ then $U_{p s s}$ is $\mathrm{U}-\mathrm{NL}$, where the membership functions $\left(M F_{i}\right)$ is defined as follows: $M F_{j} \in\{N L, N, Z, P$ and $P L\}$ as in the static fuzzy case. However, the output space has 25 different fuzzy sets. To accommodate the change in operating conditions, the adaptation algorithm changes the parameters of the input and output fuzzy sets.

The algorithm presented in this section is designated to optimize the rule base of the fuzzy controller by shifting and/or modifying the support of the input and output fuzzy sets. In addition, it will not modify the rules or the structure of the fuzzy controller. In general the membership grade of fuzzy sets $\left(\mu_{j}^{(i)}\right)$ used in this research for input or output variables has a triangular or trapezoidal configuration. These fuzzy sets are defined as follows:

In the case of a triangle $M F$, the three parameters $\{a, b, c\}$ are defined as follows:

$$
\mu_{j}^{(i)}(x ; a, b, c) \stackrel{\operatorname{def}}{=}\left\{\begin{array}{cc}
0 & x \leq a \\
\frac{x-a_{j}^{(i)}}{b_{j}^{(i)}-a_{j}^{(i)}} & a \leq x \leq b \\
\frac{c_{j}^{(i)}-x}{c_{j}^{(i)}-b_{j}^{(i)}} & b \leq x \leq c \\
0 & c \leq x
\end{array}\right.
$$

Where $a_{j}^{(i)}, b_{j}^{(i)}, c_{j}^{(i)} \in \mathbb{R}$ and $a_{j}^{(i)} \leq b_{j}^{(i)} \leq c_{j}^{(i)}$, where $\mathbb{R}$ is the real number set. This means that $\mu_{j}^{(i)}\left(a_{j}^{(i)}\right)=0, \mu_{j}^{(i)}\left(b_{j}^{(i)}\right)=1$ and $\mu_{j}^{(i)}\left(c_{j}^{(i)}\right)=0$, as shown in Fig. 12. The triangular mem-

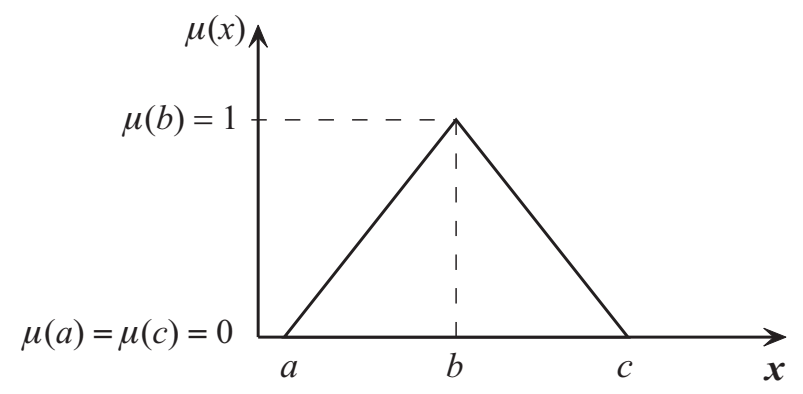

Fig. 12: Triangular membership function $(M F)$ bership functions used symmetric consequents and antecedents to represent the fuzzy set [11].

In the case of a trapezoidal $M F$, the four parameters $\{a, b$, $c, d\}$ are defined as follows:

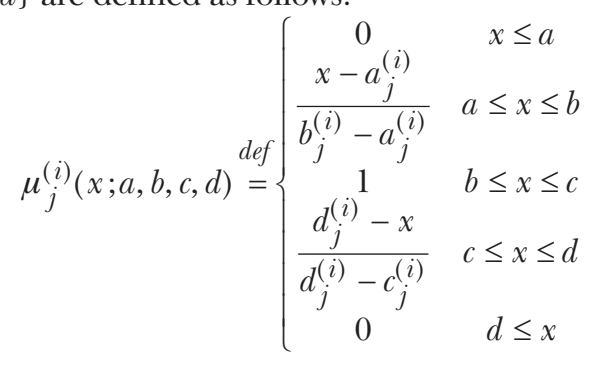

Where $a_{j}^{(i)}, b_{j}^{(i)}, c_{j}^{(i)}, d_{j}^{(i)} \in \mathbb{R}$ and $a_{j}^{(i)} \leq b_{j}^{(i)} \leq c_{j}^{(i)} \leq d_{j}^{(i)}$. This means that $\mu_{j}^{(i)}\left(a_{j}^{(i)}\right)=0, \mu_{j}^{(i)}\left(b_{j}^{(i)}\right)=1, \mu_{j}^{(i)}\left(c_{j}^{(i)}\right)=1$ and $\mu_{j}^{(i)} d\left(c_{j}^{(i)}\right)=0$, as shown in Fig. 13. The trapezoidal membership functions used symmetric consequents and antecedents to represent the fuzzy set [11].

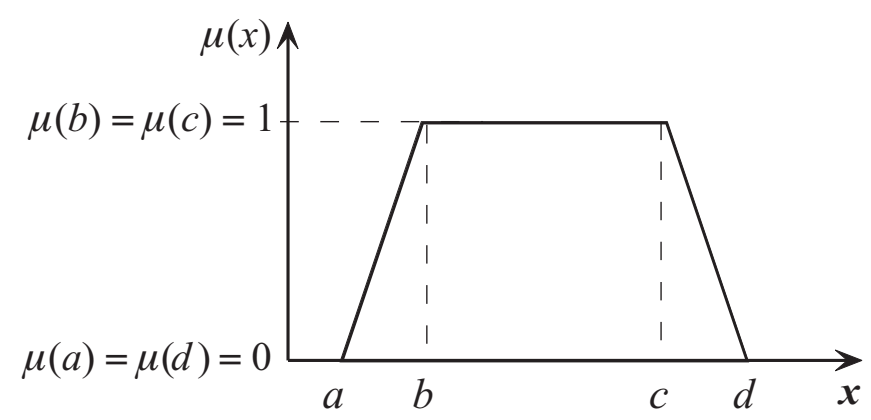

Fig. 13: Trapezoidal membership function $(M F)$

The updated parameters of the membership functions of the fuzzy set for the input and output variables used the on-line back-propagation (BP) algorithm [12]. A simple mathematical form of the on-line updating for the $M F$ is given by the following equation:

$$
\theta\{k+1\}=\theta\{k\}+\alpha \times \Delta \theta\{k\}
$$

Where $\alpha$ represents the learning rate and $\theta$ represents the parameters $a_{j}^{(i)}, b_{j}^{(i)}, c_{j}^{(i)}, d_{j}^{(i)} \in \mathbb{R}$ of the membership function of the fuzzy sets for the input and output variables. $\Delta \theta\{k\}$ is the change of these parameters based on the performance of the system under study [12]. Figs. 7, 8, and 9 show the simulation results of the normalized $M F s$ of the input and output fuzzy sets before and after adaptation. The tuning strategy goal for the AFS is to achieve a fast dynamic response, with no overshoot and negligible steady state error. The complexity of the fuzzy logic controller is reduced due to the lower number of rules and adaptation of the membership function parameters.

\section{Simulation results of S.G equipped with FLC-PSS based on FFS and AFS}

The system data given in Appendix-1 is used to test the proposed FLC-PSS. The proposed FLC has two input variables, $\Delta \omega$ and $\Delta \omega^{\prime}$. Different simulation results have been obtained for the system given in Fig. 1. These results are 
obtained when the S.G. is subjected to different dynamic disturbances, while the system is normally loaded and 0.65 p.u. active power and 0.45 p.u. reactive power are delivered. These disturbances are applied as a step change in the mechanical power command and the voltage reference command, respectively.

\subsection{Mechanical torque disturbance}

The first case was studied when the S.G. was subjected to a $10 \%$ step increase in the reference mechanical torque and then the torque returned to the initial condition. Figs. 14a, b show the simulation results for this case with the power angle $\delta$, in radians, and the speed deviation $\Delta \omega$, in $\mathrm{rad} / \mathrm{sec}$.

The dynamic responses using a CPSS FLC with static seven and five fuzzy sets for the input and output variables and an adaptive fuzzy logic controller (AFLC) are shown in Figs. 14a and 14b, respectively. The dynamic response when using AFLC is superior to the three other controllers as regards the rising time, settling time and damping coefficient of the overall system. Figs. 9, 10 and 11 show the normalized

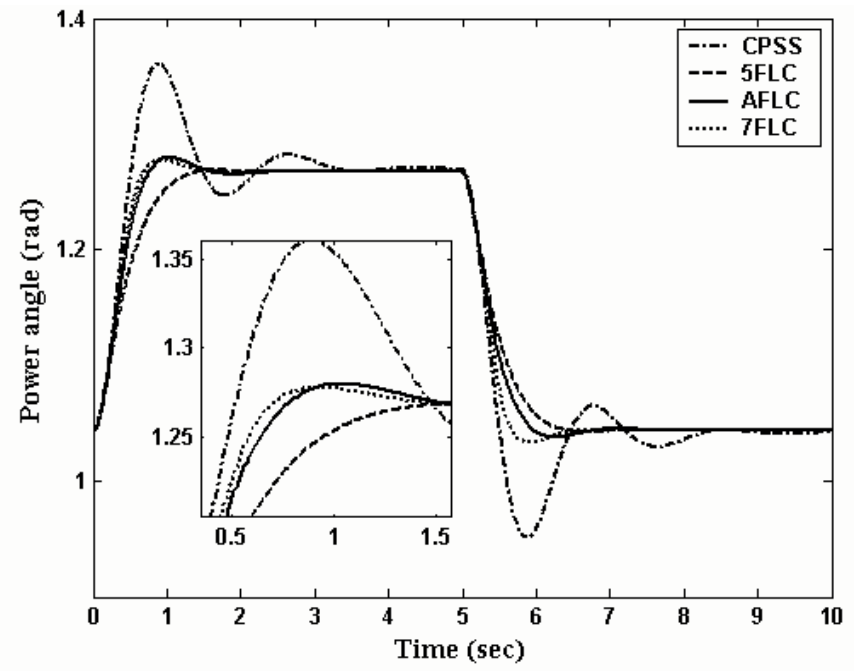

a)

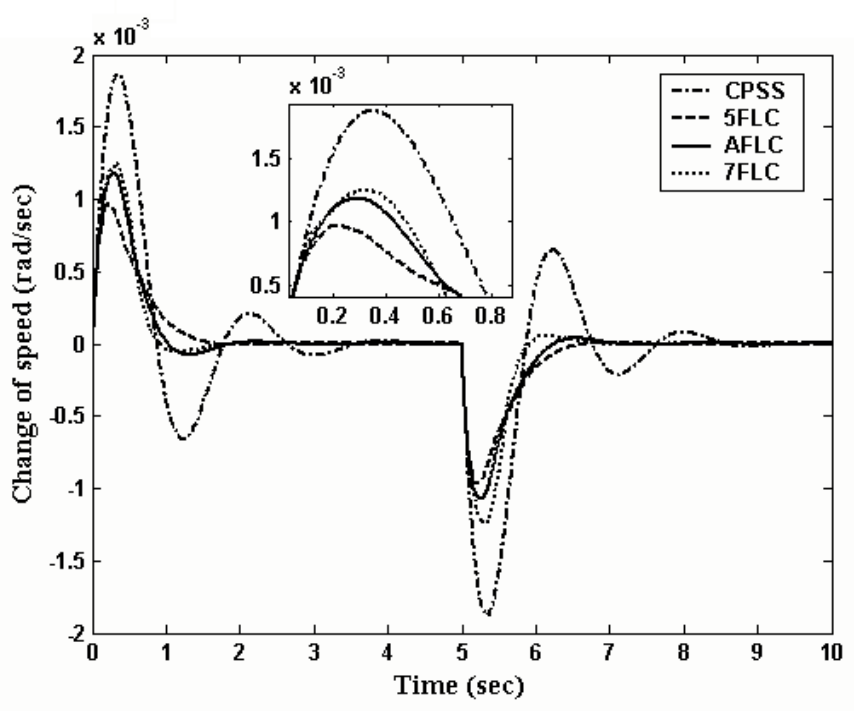

b)

Fig. 14: Dynamic response of the synchronous generator (S.G.) equipped with FLC-PSS, AFLC-PSS and CPSS, (a) Power angle displacement, (b) Speed change
MFs before and after training, using the BP technique for the input and output variables of the fuzzy controller.

\subsection{Terminal voltage disturbance}

The second case of disturbance is carried out when the S.G. is subjected to a $10 \%$ step increase in the Voltage reference, and then returns to the initial condition. Figs. 15a and $15 \mathrm{~b}$ show $\delta$, in radians, and $\Delta \omega$, in $\mathrm{rad} / \mathrm{sec}$, respectively. The dynamic performance of AFLC-PSS is much better in compared with five FLC-PSS, and the same conclusions have been reached as those obtained with Torque reference disturbances, as regards rising time, percentage overshoot, and oscillations.

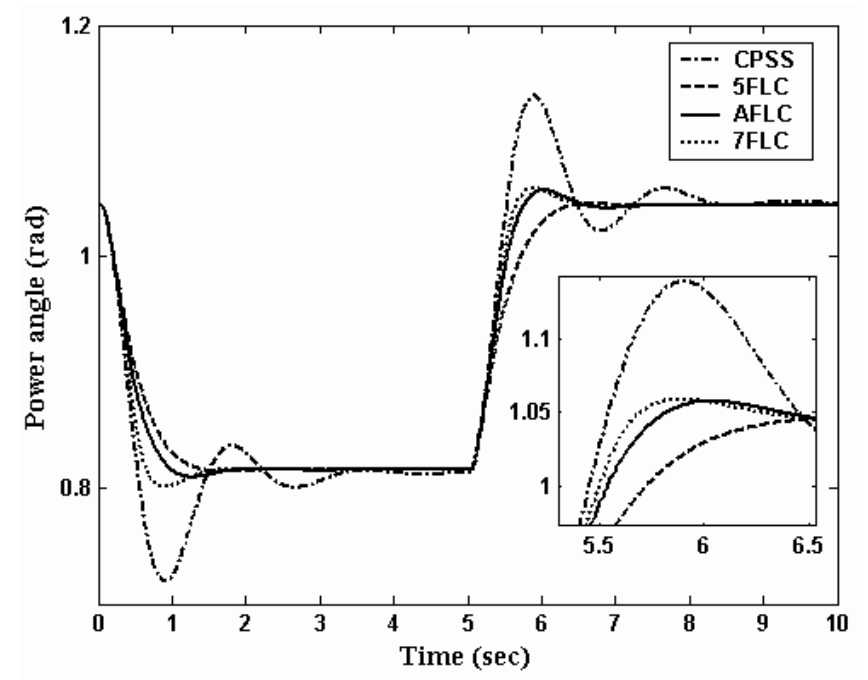

a)

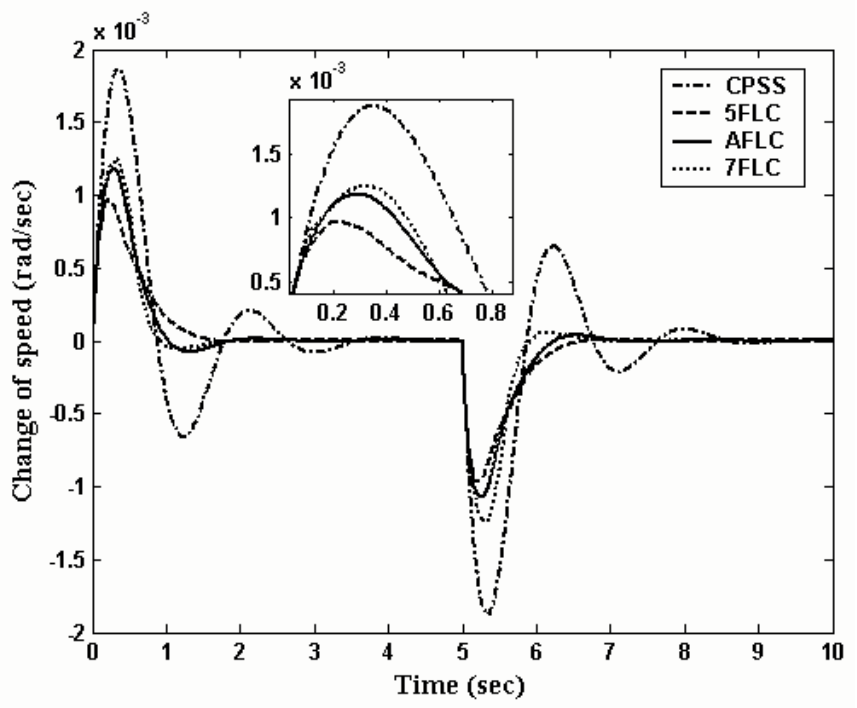

b)

Fig. 15: Dynamic response of the synchronous generator (S.G.) equipped with FLC-PSS, AFLC-PSS and CPSS, a) Power angle displacement, b) Speed change

\section{CONCLUSIONS}

This paper introduces a novel application FLC-PSS and tests it through a simulation program based on an adaptive fuzzy set. A classical fuzzy logic controller, using a fixed fuzzy set, was simulated and tested. The settling time and the rise 
time are decreased when using the adaptive fuzzy controller. A AFLC also improves the damping coefficient of the overall system under study. Simulation results show the superiority of the adaptive fuzzy controller in over other controllers. The simulation results also show the effectiveness of the proposed FL with an adaptive fuzzy set scheme as a promising technique. In addition the proposed technique reduces the computation time of the FLC. The number of rules is reduced from 49 in the case of seven fuzzy sets, to 25 when using AFLC.

\section{Appendix 1}

The data of the machine, AVR exciter and governor are given as follows:

All parameters and data are given in per-unit values

$P_{e}=0.65, \quad Q_{e}=0.45, \quad V_{t}=1, \quad X_{d}=1.2$,

$X_{d}^{\prime}=0.19, \quad X_{q}=0.743, \quad H=4.63, \quad T_{d}^{\prime}=7.76, \quad D=2$,

Local load data: $\quad G_{2}=0.249, \quad B_{1}=0.262$

Line data: $\quad R_{T . L}=0.034, X_{T . L}=0.997$

AVR data: $\quad K_{A}=400, \quad T_{A}=0.02$

Speed Governor parameters (in p.u.) $a=-0.001238$,

$b=-0.17$,

$T_{g}=0.03$.

\section{Reference}

[1] Larsen, E. V., Swann D. A.: “Applying Power System Stabilizers: Part I, Part II and Part III", IEEE Trans. on Power Apparatus \& Systems, 1981, PAS-100, p. 3017.

[2] Kothari, M. L., Nanda, J., Bhattacharya K.: "Discrete Mode Power System Stabilisers", IEE proceedings part C, 1993, 140, (6), p. 523-531.

[3] Aldeen, M., Chetty M.: “A Dynamic Ouput Feedback Power System Stabiliser", Proceedings of the Control '95 Conference, Vol. 2 (1995), Melbourne, p. 575-579.

[4] Samarasinghe, V. G. D. C., Pahalawaththa, N. C.: "Design of Universal Variable-Structure Controller for Dynamic Stabilization of Power Systems", IEE Proceedings Generation, Transmission and Distribution, Vol. 141 (1994), No. 4, p. 363-368.

[5] Anderson, P. M., Fouad, A. A.: Power System Control and Stability. IEEE press, New York, 1994.

[6] Ahmed, S. S., Chen, L., Petroianu, A.: "Design of Suboptimal $\mathrm{H}_{\infty}$ Excitation Controllers", IEEE Transactions on Power Systems, Vol. 11 (1996), No. 1, February 1996, p. 312-318.

[7] Ohtsuka, K., Taniguchi, T., Sato, T., Yokokawa, S., Ueki, Y.: "A $\mathrm{H}_{\infty}$ Optimal Theory-Based Generator Control System”, IEEE Transactions on Energy Conversion, Vol. 7 (1992), No. 1.
[8] El-Metwally, K. A., Malik, O. P.: “A Fuzzy Logic Power System Stabiliser", IEE proc. Generation, Transmission and Distribution, Vol. 145 (1995), No. 3, p. 277-281.

[9] Lie, T. T, Sharaf, A. M.: “An Adaptive Fuzzy Logic Power System Stabiliser”, Electric Power System Research, Vol. 38 (1996), p. 75-81.

[10] Hsu, Y. Y., Chen, C. R.: “Tuning of Power System Stabiliser Using Artificial Neural Network", IEEE Trans. Energy Conversion, Vol. 6 (1991), No. 4, p. 612-619.

[11] Lee, C. C.: "Fuzzy Logic Control Systems: Fuzzy Logic Controller, Part I", IEE Trans. Syst. Man, Cybernetic, Vol. 20 (1990), March /April, p. 404-418.

[12] Nürnberger, A., Nauck, D., Kruse, R.: "Neuro-Fuzzy Control Based on the NEFCON-Model", recent developments. Soft Computing Vol. 2 (1999), Springer-Verlag 1999, p. 168-182.

[13] Demello, Laskowski, T. F.: "Concepts of Power System Dynamic Stability” IEEE Trans. on Power Apparatus \& Systems, Vol. 94 (1979), p. 833.

[14] Soliman, H. F., Badr, M. A., Hellal, M. N. A.: "Improving the Performance of the Power System Stabilizer Using a Variable Rule Based Fuzzy Logic Controller", Scientific Bulletin of Ain Shams Univ., Vol. 39 (2004), No. 3, September 2004, Egypt.

Associate Prof. Dr. Ing. Hussein F. Soliman

e-mail: hfaridsoliman@yahoo.com

Dept. of Electric Power and Machine

Faculty of Engineering

Ain-Shams University

Abbasia, Cairo, EGYPT

Dr. Ing. Abdel-Fattah Attia

phone: +2025560046

fax:+2025548020

e-mail: attiaa1@yahoo.com

Astronomy Department

National Research Institute of Astronomy and Geophysics (NRIAG),

11421 Helwan

Cairo, EGYPT

Ing. Mohammed Hellal

South Cairo Electric Power Station

Helwan, Cairo, EGYPT

Prof. Dr. Ing.M. A. L. Badr

Dept. of Electric Power and Machine

Faculty of Engineering

Ain-Shams University

Abbasia, Cairo, EGYPT 